\title{
Dynamic Conditional Correlation GARCH: A Multivariate Time Series Novel using a Bayesian Approach
}

\author{
Diego Nascimento \\ University of Sao Paulo, dnstata@gmail.com \\ Cleber Xavier \\ University of Sao Paulo, cleber.statistics@gmail.com \\ Israel Felipe \\ Federal University of Ouro Preto, israeljfelipe@gmail.com \\ Francisco Louzada Neto \\ University of Sao Paulo, louzada@gmail.com
}

Follow this and additional works at: https://digitalcommons.wayne.edu/jmasm

Part of the Applied Statistics Commons, Social and Behavioral Sciences Commons, and the Statistical Theory Commons

\section{Recommended Citation}

Nascimento, D., Xavier, C., Felipe, I., \& Neto, F. L. (2019). Dynamic conditional correlation GARCH: A multivariate time series novel using a Bayesian approach. Journal of Modern Applied Statistical Mehtods, 18(1), eP2722. doi: 10.22237/jmasm/1556669220

This Regular Article is brought to you for free and open access by the Open Access Journals at DigitalCommons@WayneState. It has been accepted for inclusion in Journal of Modern Applied Statistical Methods by an authorized editor of DigitalCommons@WayneState. 
Dynamic Conditional Correlation GARCH: A Multivariate Time Series Novel using a Bayesian Approach

\section{Cover Page Footnote}

The research was partially supported by CNPq, FAPESP and CAPES of Brazil. 


\section{Dynamic Conditional Correlation GARCH: A Multivariate Time Series Novel using a Bayesian Approach}

\author{
Diego Nascimento \\ University of São Paulo \\ São Paulo, Brazil

\section{Israel Felipe} \\ Federal University of Ouro Preto \\ Ouro Preto, Brazil
}

\author{
Cleber Xavier \\ University of São Paulo \\ São Paulo, Brazil \\ Francisco Louzada Neto \\ University of São Paulo \\ São Paulo, Brazil
}

The Dynamic Conditional Correlation GARCH (DCC-GARCH) mutation model is considered using a Monte Carlo approach via Markov chains in the estimation of parameters, time-dependence variation is visually demonstrated. Fifteen indices were analyzed from the main financial markets of developed and developing countries from different continents. The performances of indices are similar, with a joint evolution. Most index returns, especially SPX and NDX, evolve over time with a higher positive correlation.

Keywords: $\quad$ DCC-GARCH, Bayesian model, visual data mining, financial contagion

\section{Introduction}

International economy studies involve aspects related to economic activity among nations and its rules of operation, legal aspects, monetary exchanges, flows of goods and services (Eichengreen et al., 1996). Among with the phenomenon of globalization, more interaction and interdependence among nations makes pressing the perfect functioning of these relations. In this sense, the commonly adopted solution is the creation of intercontinental and supranational organizations that intercede in order to balance these relations and promote the gross domestic product of all participants.

One way to verify the interaction and interdependence among nations empirically is to relate the performance of an economy to the evolution of its

doi: 10.22237/jmasm/1556669220 | Accepted: November 30, 2017; Published: February 25, 2020.

Correspondence: Diego Nascimento, dnstata@gmail.com 


\section{NASCIMENTO ET AL}

financial markets by associating the performance of macroeconomic variables (level of economic activity, interest rate, exchange rate etc.) and asset prices. In theoretical terms, we can highlight the works (Blanchard, 1990; Hansen \& Singleton, 1983; Campbell \& Ammer, 1993; Dornbusch \& Fischer, 1980), in which all observed some kind of relation between the evolution of the financial market and the respective macroeconomic variables. As an example, Blanchard (1990) analyzed the relationship between asset prices and economic activity, in order to characterize the interaction between the variables and verify the joint hypothesis regarding possible changes in fiscal and monetary policies.

Because economic representation can take place through the performance of the respective markets, one can seek evidence of dependencies between nations through the interrelationship between their asset markets. Thus, there is a need for the creation of financial assessment instruments that demonstrate the links between economies. Due to the emergence of large volumes of global transactions, the financial sector sought to promote multilateral agreements aimed at controlling these negotiations more effectively. The trend towards deepening the association of domestic capital with international financial markets is increasingly evident in all economies (Dambrós, 2014).

Data collected from fifteen nations observed the respective problems: (1) presence of high dimensionality in the data, (2) different lengths between the series analyzed, and (3) the definition of similarity, in relation to pattern recognition, differs in Time Series, as explicit (Bettaiah \& Ranganath, 2014). To solve these problems, it is necessary to expand the classic models, incorporating the idea of Big Data. These models may be widely applicable in the fields of finance, environment, engineering, biological, medical sciences etc.

The evolution of these data can be visualized through a hypercube considering the dimensions of time and space. This type of analogy facilitates the understanding of the various visualization techniques with temporal dependence, whose domain of data can be analyzed in terms of elementary operations and be adapted to other domains and problems (Bach et al., 2014). These authors also add a critical look at the non-standardization of nomenclatures for the techniques, which generate equivalent work without adding much to the problem of dimensionality reduction in Time Series.

Creating a data view requires a series of questions about the different encodings used to facilitate the understanding of data types, such as numbers, categories or networks. It is necessary to identify the appropriate data and select the effective visual encodings to map the values of the data pertaining to graphic characteristics, such as position, size, shape and color. The challenge is that for any 


\section{DCC-GARCH UNDER THE BAYESIAN APPROACH}

data set the number of visual encodings and therefore the space of possible visualization drawings is extremely large. Thus, information visualization seeks to reproduce a high-dimensional data layout in a version that is representative and more efficient for analysis. Some techniques such as Parallel Coordinates, ScatterPlot Matrix, Glyphplot, Andrew's Plot, forced-based can be used for this purpose (Telea, 2014).

The techniques of visualization of Multivariate Time Series seek mainly to address the issue of the reduction of high dimensionality, as for example the graphs Index Chart, stream graph, small multiplex and horizon graphs. There has been a great increase in the number of methodologies, techniques and tools aimed at this type of visualization. However, many of them perform similar procedures, presenting only different nomenclatures. Some solutions for dimensionality reduction can be conducted through user interaction, making cuts in the database, making selections to guide the data visualization (Müller \& Schumann, 2003; Luo et al., 2010). Visualization tools treat data in a rather general way, being able to handle a wide variety of temporal data, at the cost of losing the capacity for indepth analysis of the data.

\section{State of the Art}

Time series are sets of records with their values collected over time, so the size of time series grows over time (Ratanamahatana et al., 2005). Much of the data found in the real world has a temporal component or can be analyzed in a temporal way (Javed \& Elmqvist, 2010). This type of data occurs in many domains, such as finance and business, health, meteorology, astronomy. The more the size and complexity of time series data, the greater the challenges and requirements for analyzing them (Cho et al., 2014).

\section{Time Series Visualization}

Visual perception is the ability to graphically interpret the information present in the data. Therefore, the need to discover patterns in large bases of time series requires researchers to develop visualization tools to acquire knowledge about the data. However, modern visual analysis techniques are still strongly based on traditional visualization techniques, such as line graphs, histograms and scatter plots (Heer et al., 2010). In fact, one of the simplest and most popular ways to visualize time series data is line graphing. However, viewing multiple time series at the same time in a line chart can be difficult, due to the limited space and 


\section{NASCIMENTO ET AL}

resolution of computer screens, which can cause visual disorder (Perin et al., 2013). Representing billions of temporal records in millions of pixels is a major challenge in the area of Information Visualization (Shneiderman, 2008). While line graphs deal fairly effectively with some time series, common tasks within this data domain often involve multiple time series evolving simultaneously (Javed et al. 2010).

A comprehensive review on time series visualization was presented, setting out criteria and comparing the various visual metaphors used in the analysis of the topic (Aigner et al., 2008). Viewing multiple series in a small space has led researchers to the development of tools and representation techniques that allow analyzing the relationships between data from different categories (Krstajic et al., 2011). Another important aspect of this data is the dynamics of its information flow, which requires the visualization to be updated incrementally.

\section{Volatility and Financial Returns}

It is a great of interest, especially in Economics, the study of volatility and returns in a time series. Consider $P_{t}$ as being the price of an asset at time $t$ and $p_{t}$ as the $\operatorname{logarithm}$ of the price of this asset, i.e., $p_{t}=\log \left(P_{t}\right)$. From this we can define a measure known in the literature by feedback at time $t$ to be

$$
y_{t}=\log \left(\frac{P_{t}}{P_{t-1}}\right)=p_{t}-p_{t-1} .
$$

Autoregressive Conditional Heteroskedasticity (ARCH) models are a form proposed by Engle (1982) to study the behavior of financial returns. In this type of approach, it is considered that the volatility (conditional variance) at an instant $t$ does not depend on the past returns. In this way, Bollerslev (1986) proposed a generalization for the $\mathrm{ARCH}$ models in which it considers that the volatility depends both on the past values of the returns and the past values of the volatility. These models are known in the literature as GARCH (Generalized ARCH).

Subsequently, several multivariate extensions were proposed in the literature. Among these extensions we can highlight the CCC-GARCH (Constant Conditional Correlation GARCH) model proposed by Bollerslev (1990) in which the correlation between the series of returns is considered constant over time. Another currently used extension is the DCC-GARCH (Dynamic Conditional Correlation GARCH) model proposed by Engle (2002) and Tse and Tsui (2002), which considers the correlation between the variant volatilities over time. 


\section{DCC-GARCH UNDER THE BAYESIAN APPROACH}

\section{Multivariate Time Series Models}

Consider $\mathbf{y}_{t}=\left(y_{t 1}, \ldots, y_{t m}\right)^{\prime}$ as a vector of returns at time $t$ for $m$ time series. Therefore, we can define multivariate GARCH models as being

$$
\mathbf{y}_{t}=\mathbf{H}_{t}^{1 / 2} \boldsymbol{\varepsilon}_{t}
$$

where $\mathbf{H}_{t}$ is the matrix of conditional covariance and $\mathbf{H}_{t}{ }^{1 / 2}$ is a definite positive matrix $m \times m$. The vector of errors $\boldsymbol{\varepsilon}_{t}$ has order $m \times 1$ and has mean and variance given by $\mathrm{E}\left(\boldsymbol{\varepsilon}_{t}\right)=0$ and $\operatorname{Var}\left(\boldsymbol{\varepsilon}_{t}\right)=\mathbf{I}_{m}$, where $\mathbf{I}_{m}$ is an identity matrix of order $m$. In the literature there are several ways to specify the $\mathbf{H}_{t}$ matrix. Described as

$$
\mathbf{H}_{t}=\mathbf{D}_{t} \mathbf{R}_{t} \mathbf{D}_{t}
$$

Then

$$
\begin{aligned}
& \mathbf{D}_{t}=\operatorname{diag}\left(h_{11, t}^{1 / 2}, \ldots, h_{m m, t}^{1 / 2}\right), \\
& \mathbf{R}_{t}=\operatorname{diag}\left(\mathbf{Q}_{t}\right)^{-1 / 2} \mathbf{Q}_{t} \operatorname{diag}\left(\mathbf{Q}_{t}\right)^{-1 / 2}, \\
& \mathbf{Q}_{t}=(1-a-b) \mathbf{R}+a \boldsymbol{\mu}_{t-1} \boldsymbol{\mu}_{t-1}^{\prime}+b \mathbf{Q}_{t-1}
\end{aligned}
$$

where $\boldsymbol{\mu}_{t}=\mathbf{D}_{t}^{-1} \mathbf{y}_{t}, \mathbf{R}$ is the unconditional covariance matrix of $\boldsymbol{\mu}_{t}$. The parameter constraints of the additional parameters are $a>0, b>0$ and $a+b<1$. Considering $\theta$ as the set of all model parameters, we can define the joint distribution of returns as a function of the probability density chosen for the errors $\boldsymbol{\varepsilon}_{t}$.

$$
\mathrm{p}\left(\mathbf{y}_{t} \mid \mathbf{I}_{t}\right)=\left|\mathbf{H}_{t}\right|^{-1 / 2} \mathrm{p}_{\boldsymbol{\varepsilon}_{t}}\left(\mathbf{H}_{t}^{-1 / 2} \mathbf{y}_{t}\right), \quad t=1, \ldots, T
$$

being $\mathbf{I}_{t}=\left(y_{t-1}, y_{t-2, \ldots}\right)$ the information until time $t$. Therefore, we obtain the likelihood function given by

$$
\mathrm{L}(\theta)=\prod_{i=1}^{T}\left|\mathbf{H}_{t}\right|^{-1 / 2} \mathrm{p}_{\varepsilon_{t}}\left(\mathbf{H}_{t}^{-1 / 2} \mathbf{y}_{t}\right)
$$

and for the DCC-GARCH model we have 


\section{NASCIMENTO ET AL}

$$
\mathrm{L}(\theta)=\prod_{i=1}^{T}\left[\prod_{i=1}^{m} h_{i i, t}^{-1 / 2}\right]\left|\mathbf{R}_{t}\right|^{-1 / 2} \mathrm{p}_{\varepsilon_{t}}\left(\mathbf{D}_{t} \mathbf{R}_{t} \mathbf{D}_{t}^{-1 / 2} \mathbf{y}_{t}\right)
$$

Strong evidence has emerged in the literature that financial returns have a certain degree of asymmetry. One way to deal with this type of problem is to assume some degree of asymmetry in the distribution of errors. Fioruci et al. (2014) used the method proposed by Bauwens and Laurent (2005) and defined asymmetric probability distributions as the standard asymmetric and standardized distribution. Its function of probability distribution is given by

$$
\mathrm{p}\left(\mathbf{y}_{t} \mid \mathbf{I}_{t}\right)=\left(\frac{2}{\pi}\right)^{\frac{m}{2}}\left(\prod_{i=1}^{m} \frac{\gamma_{i} \sigma_{i}}{1+\gamma_{i}^{2}}\right) \exp \left\{-\frac{1}{2} \sum_{i=1}^{m} z_{i}^{* 2}\right\}
$$

Then

$$
z_{i}^{*}= \begin{cases}\left(z_{i} \sigma_{i}+\mu_{i}\right) / \gamma_{i}, & \text { if } z_{i} \geq-\mu_{i} / \gamma_{i} \\ \left(z_{i} \sigma_{i}+\mu_{i}\right) / \gamma_{i}, & \text { if } z_{i}<-\mu_{i} / \gamma_{i}\end{cases}
$$

and that $\boldsymbol{\gamma}$ is the asymmetry parameter.

For the estimation of the parameters, the function bayesDccGarch was used, and defined in 120 thousand the number of simulations with 30 thousand being the sample of heating (burn-in). The estimation procedure is based on the MetropolisHastings algorithms, an MCMC approach (Monte Carlo methods via Markov chains). For more details, see Fioruci et al. (2014). The R package bayesDccGarch proposed by Fioruci et al. was used.

\section{Methodology}

Fifteen indexes of the world stock market were analyzed: Brazil (Ibovespa), Argentina (Merval), Chile (IPSA), Mexico (MEXBOL), Peru (IGBvl), Venezuela (IBVC), South Korea (Kospi), Japan (Nikkei), Hong Kong (Hang Seng), USA (Dow Jones, S\&P500 and Nasdaq), London (FTSE 100), Frankfurt (DAX 30) and Paris (CAC 40), from JAN/2004 to OUT/2012. This period was intentionally in order to use the European indexes separately given they are all unified now. The choice of the period considered the limitations on the confidence of the information of Argentina and Venezuela, in which after this period the world bank began to 


\section{DCC-GARCH UNDER THE BAYESIAN APPROACH}

distrust the macroeconomic indexes published by the mentioned countries. This work did not consider the difference between time zones and their respective operating durations (hours of operation), analyzing only the closing values of the respective indices. The main motivation was based on the determination of elements that indicate the interrelationship between the markets.

\section{Discussion}

The performance of the indices, visually they look similar given their evolution among time. Figure 1 shows each stock market index per line, in which as their growth reaches the top restarts again with a darker color. Also, it shows that exists a mutual increase concentrated the years of 2010 and mid-2012, followed by a fall in the crisis in the European Union.

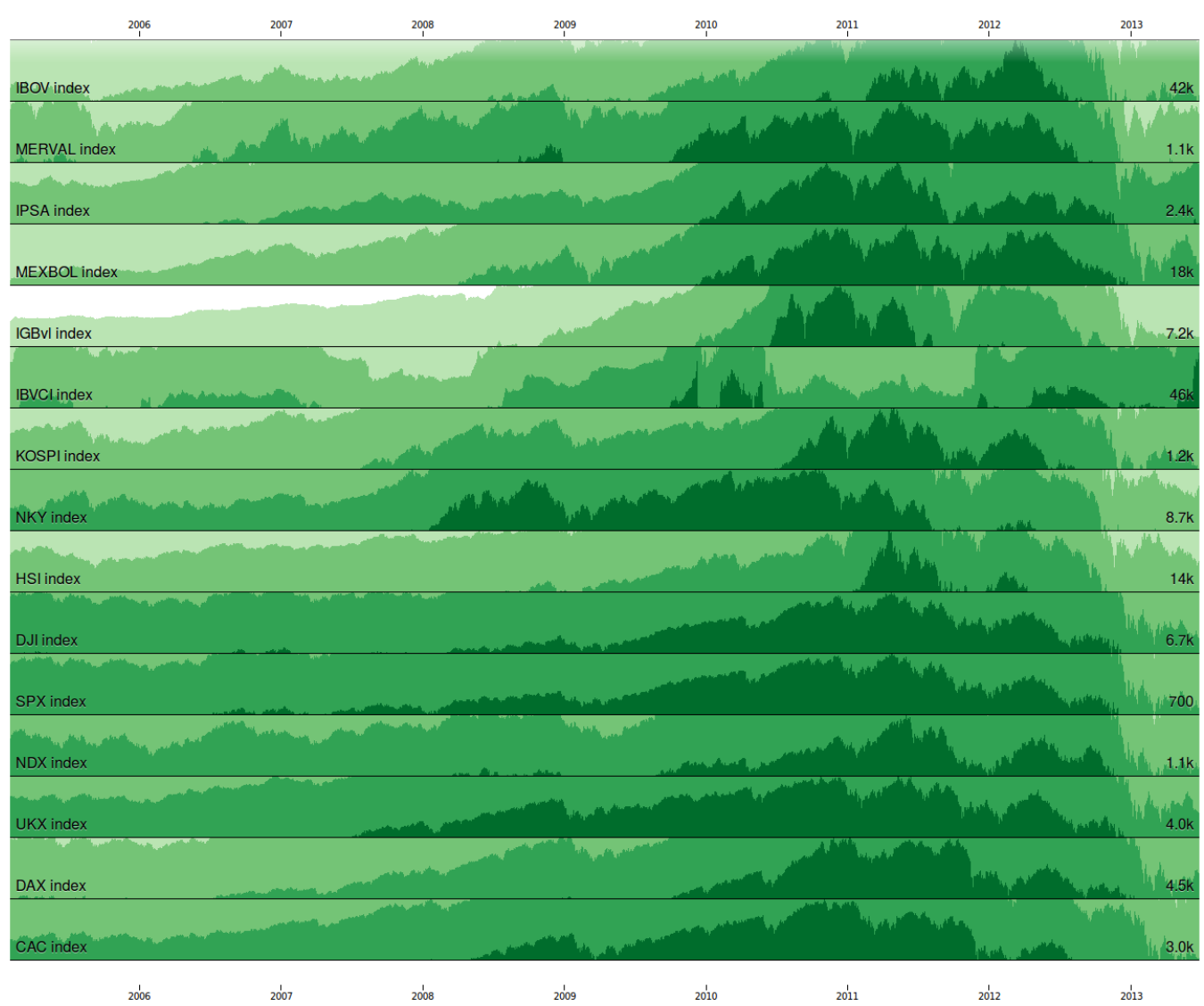

Figure 1. Plot of the 15 indices of the world stock markets 


\section{NASCIMENTO ET AL}

The daily relation between the markets was also verified, through their respective progressions, considering their respective clusters. Figure 2 exhibits the negotiations' volume, where which line represents the intraday negotiation and each column is a market. Notice the formation of pipelines showing the repeated rolling performance of the markets as well as their dependencies. This graph is called parallel coordinates.

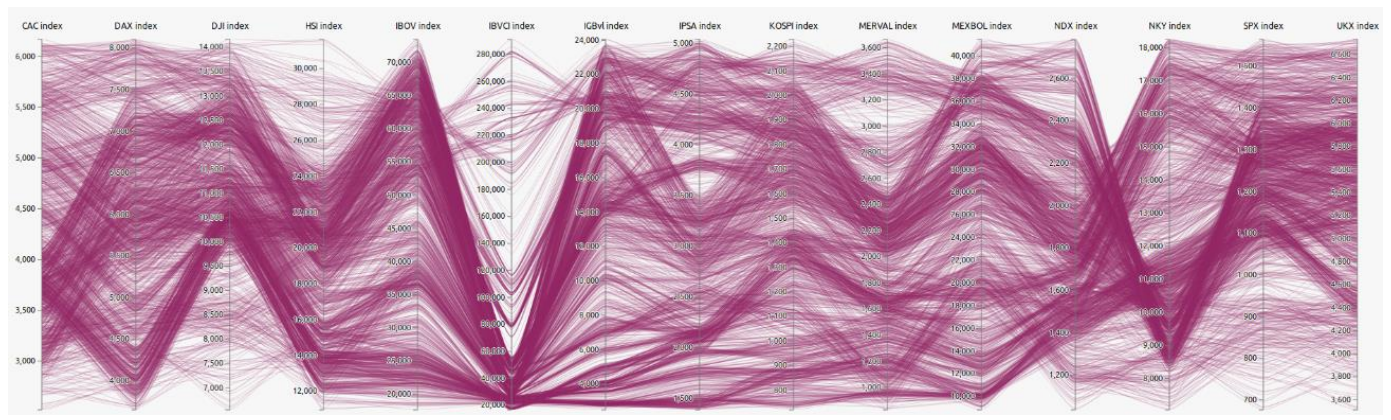

Figure 2. Parallel Coordinates of the 15 markets indices
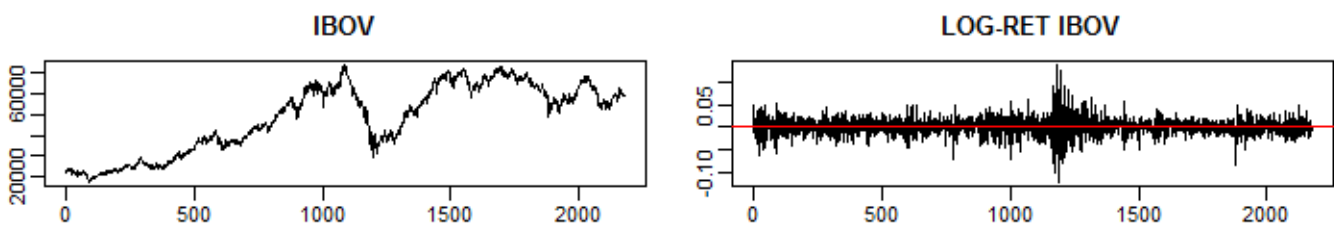

MERVAL
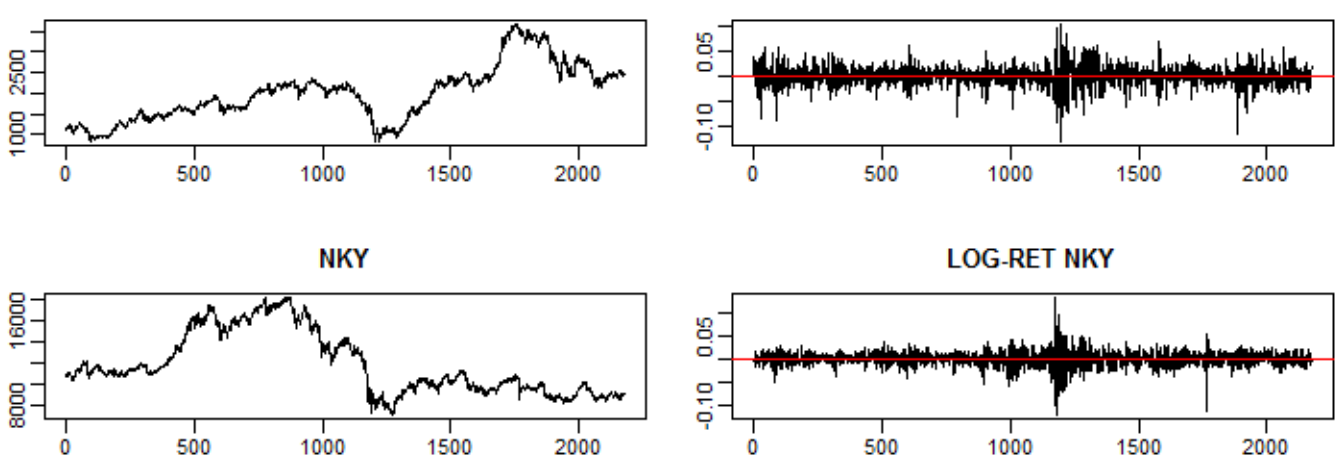

Figure 3. Plot of the original series and their transformed returns 


\section{DCC-GARCH UNDER THE BAYESIAN APPROACH}

In this way, the formation of groups between the coordinates represent the temporal dependencies, evidencing elements that lead to the belief that their performance is associated, considering the volume of operations of each market. Then, both Figures 1 and 2 show visually a noticeable joint evolution/dependence in the global market's indices.

For further analyses, the series were transformed into logarithmic' returns series. Before modeling, some hypothesis must be verified about the role of indexes' return. It was noticeable the similarity between the markets, the same way as the original series, exemplified by three series in Figure 3.

Then, in order to test the dependence hypothesis, the correlation between the market's transformed returns was analyzed, as well as, tested using Pearson test, considering a significance level of 5\%. Figure 4 shows the correlation intensity between the markets and presents X's on the markets with a statistical' absence of significance. The only market related with no other was the Venezuela Stock Market (IBVCI).

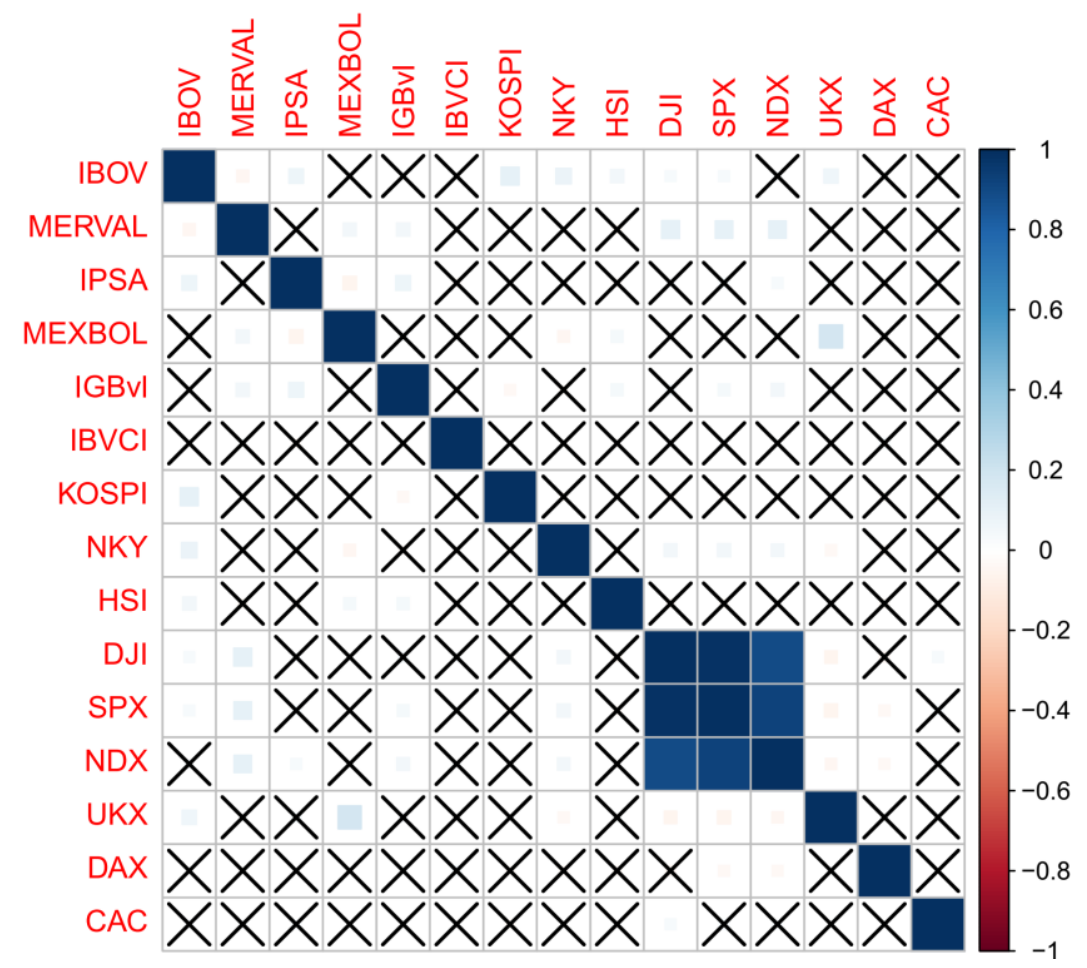

Figure 4. Correlation Plot between the markets' returns combined with Pearson's test 


\section{NASCIMENTO ET AL}

Retornos IBOV
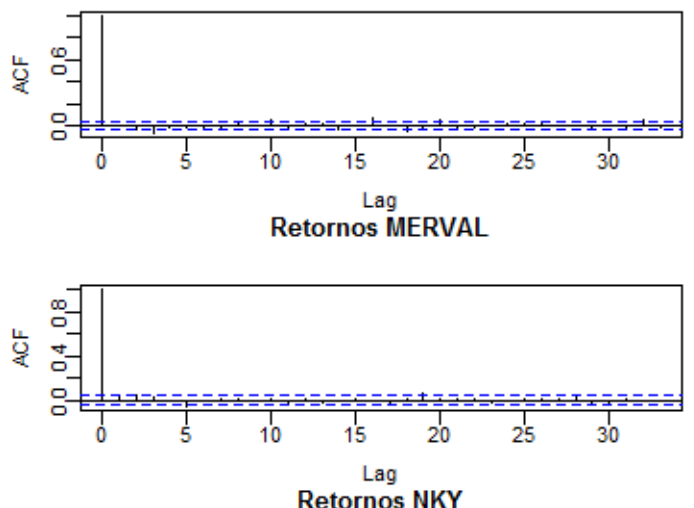

Retornos NKY

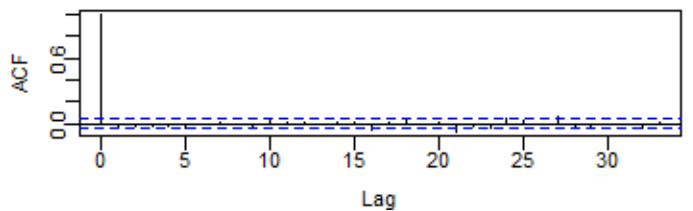

Retornos^2 IBOV
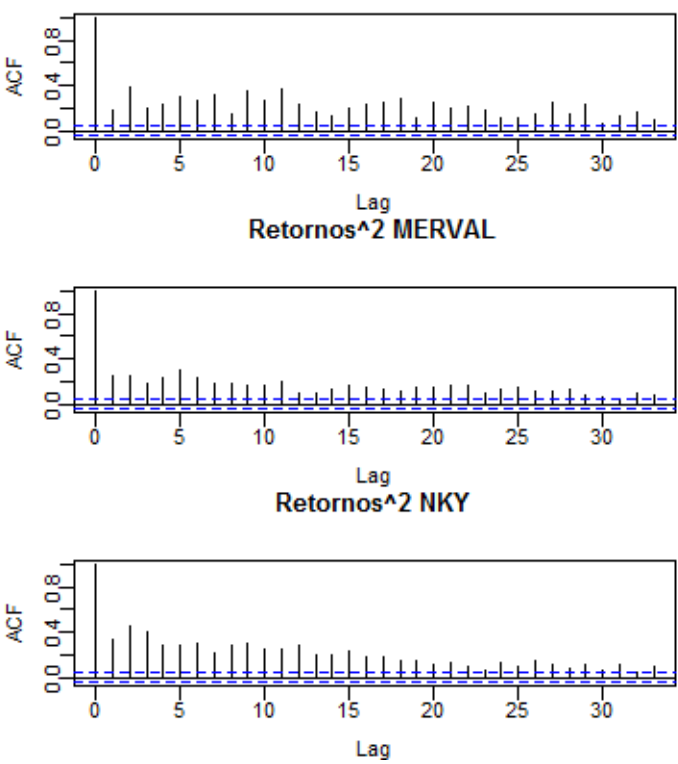

Figure 5. Plot of Autocorrelation function (ACF) of some markets' returns

The original indices' series present several violations necessary for the use of classic time series models, especially stationarity. Thus, Figure 4 shows three series, as an example, which visually it is possible to verify the presence of trend (not having constant mean in time) and mainly a non-constant variance of the series (presence of heteroscedasticity). For these reasons, there is a greater interest in modeling the returns of these series, since they will present better interpretations, whose performance is associated with a chance of return used in the decision making.

As the previous section mentioned, Dynamic Conditional Correlation GARCH (DCC-GARCH) was used to incorporate a multivariate perspective for the marginal distributions model. We consider $\operatorname{GARCH}(1,1)$ for the conditional variances in $\mathrm{Dt}$, from this the number of estimated parameters of the model will be $3 m+2$, with the number of series used during pet. Thus, using 15 series we have the total number of 47 estimated parameters. In addition, we have the parameter $\gamma_{i}$, $i=1, \ldots, m$ which represents the degree of asymmetry of the standard asymmetric and standardized distribution, with $\gamma=1$ representing its symmetric version, $\gamma<1$ its asymmetric version on the left and $\gamma>1$ its asymmetric version on the right. In this way, we had a total of 62 estimated parameters. 


\section{DCC-GARCH UNDER THE BAYESIAN APPROACH}
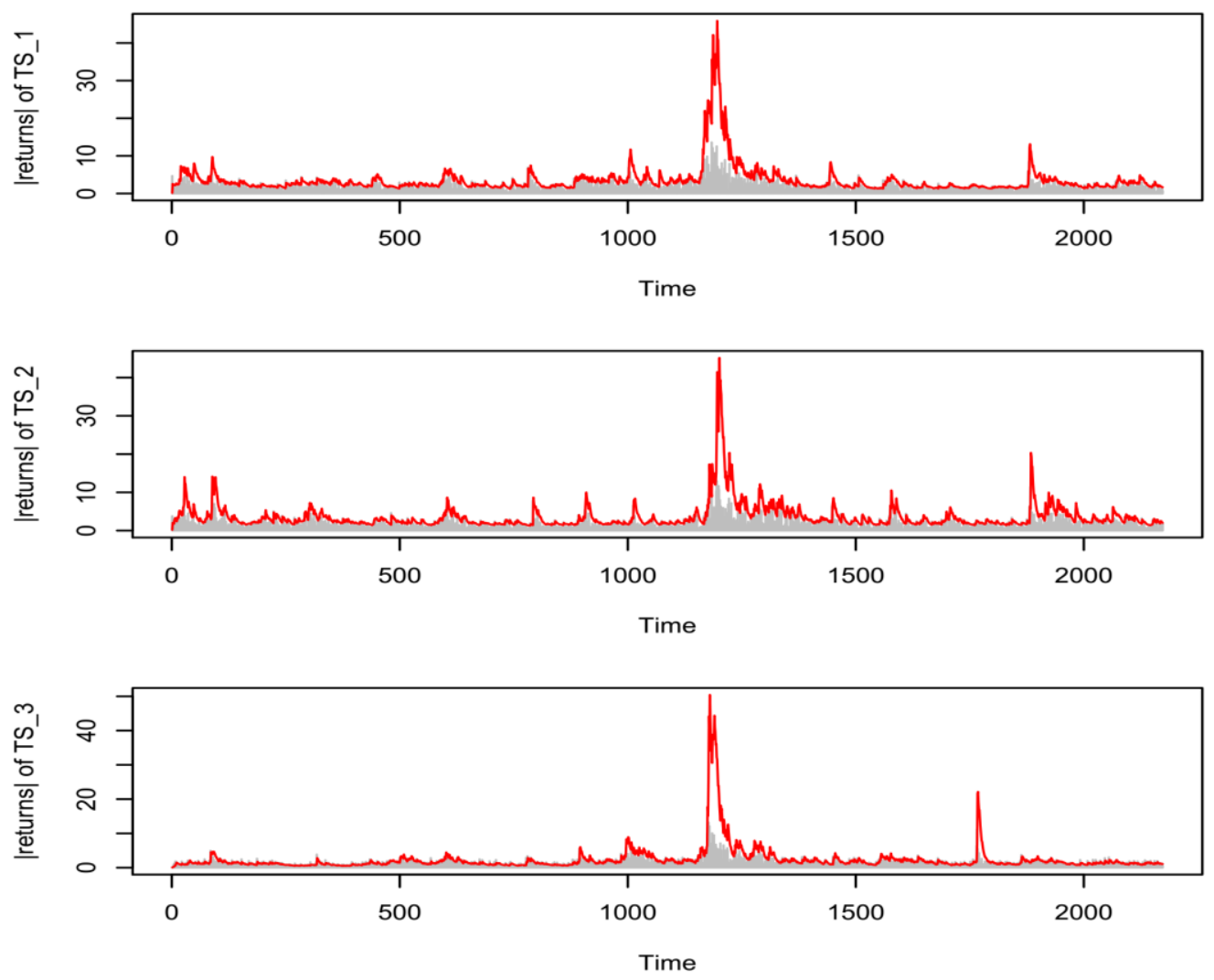

Figure 6. Plot of some markets' returns series (gray) vs model adjustment (red)

Illustrating the results, Figure 6 shows the return of the series in gray followed by the red line that represents the adopted model, which refers to showing a good fit considering also the moments of greater volatility (incorporating the peaks of the series).

The completed results are presented in a table attached in the appendix, summarized the estimations of the parameters, for the proposed model, additionally of that its associated standard errors and sample's quantile distribution. For the parameter $\gamma$ only the $\gamma_{11}$ (SPX) and $\gamma_{12}$ (NDX) present the value 1 in the range of sample quantiles. Thus, the other indexes present an asymmetric behavior.

Another important result, given the DCC-GARCH model, refers to conditioned (lagged) time correlation analysis. This relationship between the indexes can be observed with a dynamic perspective, and recalculated over time, thus updating the information incorporated at each moment. Figure 7 shows the evolution in time, they still seem to be oscillating around their respective mean. 


\section{NASCIMENTO ET AL}

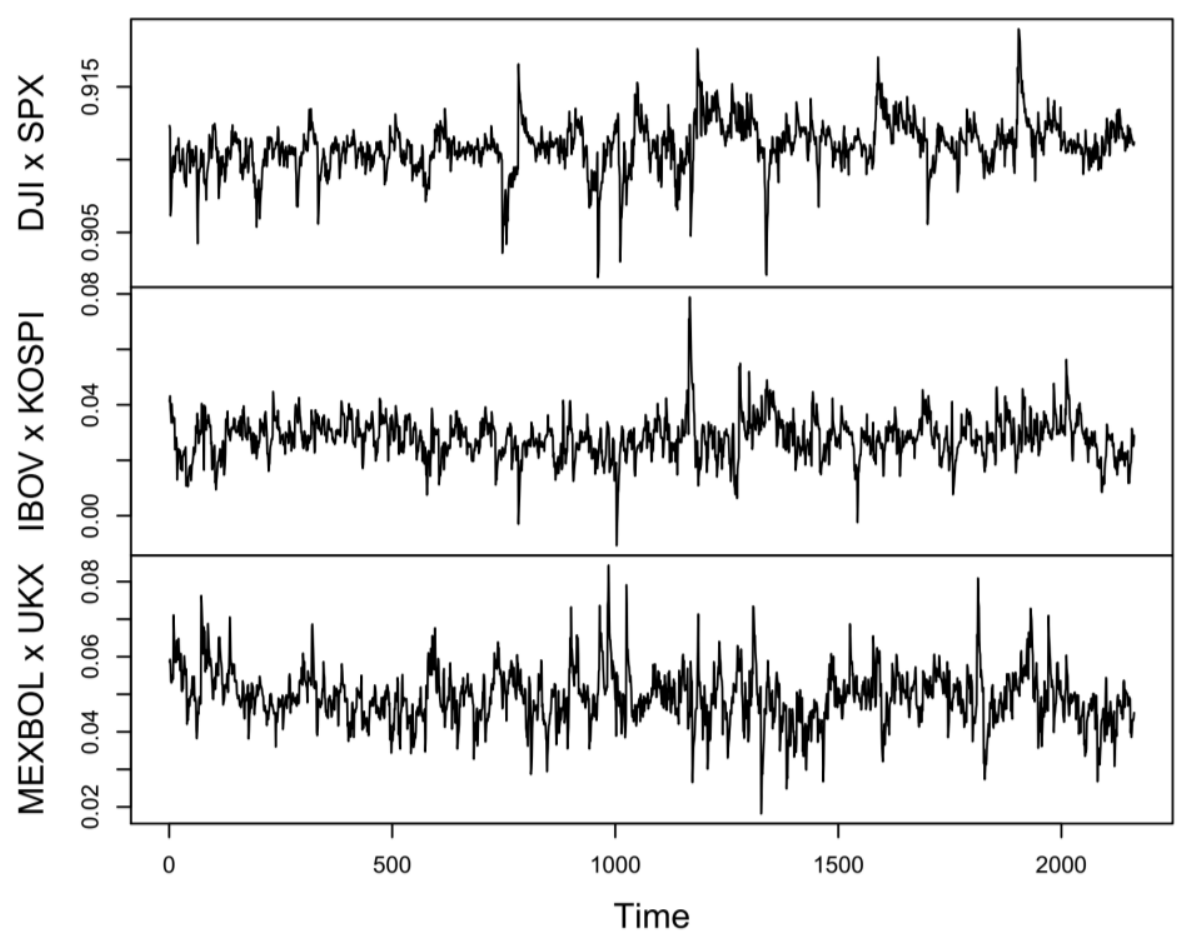

Figure 7. Dynamic conditional correlation plot of some markets' returns

Results show the DCC-GARCH incorporated well the temporal dependence, concomitantly the interrelation among the financial markets verifying empirically their relations.

\section{Conclusions and Implications}

One of the generalizations of GARCH models, Dynamic Conditional Correlation GARCH (DCC-GARCH) was considered, which assumes the conditional correlation matrix changes over time, making them parsimonious. This change is determined by the inclusion of only two parameters, facilitating the inference and prediction of the model in the context of multivariate series. The Bayesian approach has been increasing in the last years due to the growth of computational capacity, and shows some advantages, such as a better interpretation and incorporation of prior information to parameters. 


\section{DCC-GARCH UNDER THE BAYESIAN APPROACH}

Presented as one possible application of this type of modeling, there is a growing concern about studies on the behavior of global financial markets. In this sense, research that adds new tools or methodologies that can advance in the studies already produced can offer relevant contributions to the improvement of the financial relations concept of world markets. This paper made a good contribution, especially when considering the conditional analysis of correlation through a dynamic view. The temporal dependence of returns on world market indices was considered here to promote an empirical reflection on the measurement of the stock indices of fifteen world markets under the Bayesian approach. The performances of world indices are similar, with a considerable increase in 2010 and 2012 (joint evolution). The dependence and relationship between markets were confirmed, which suggests the performances invested countries are associated. Finally, most of the index returns, especially the SPX and NDX, evolve in time with a higher positive correlation, oscillating concomitantly around their respective conditional tendencies.

The obtained results reveal important questions about the relationship between conception and methodology. The concept of contagion used in this study - defined in this study as the statistical breakdown in the computed DCCs, according to the measurement presented by the structural changes in the medians and medians of the indices - allows the use of the correlation of returns to be used as a proxy to investigate the interrelations between the observed world economy.

A limitation of this work is the lack of scientific tools to precisely identify the transmission channels of shocks, which need to be investigated further. According to Eichengreen et al. (1996), this type of analysis is relevant because it allows deeper investigations of the causes of the contagion effect, as well as providing space for discussions about the fragility of the markets vis-à-vis the international economy of the countries. It is important to highlight these results on on financial contagion at the level of political-economic discussion, especially for decisionmakers, investors and policymakers.

\section{References}

Aigner, W., Miksch, S., Muller, W., Schumann, H., \& Tominski, C. (2008). Visual methods for analyzing time-oriented data. IEEE Transactions on Visualization and Computer Graphics, 14(1), 47-60. doi: 10.1109/TVCG.2007.70415 


\section{NASCIMENTO ET AL}

Bach, B., Dragicevic, P., Archambault, D., Hurter, C., \& Carpendale, S. (2014). A review of temporal data visualizations based on space-time cube operations. In R. Borgo, R. Maciejewski, \& I. Viola (Eds.), Eurographics conference on visualization (pp. 1-19).

Bauwens, L., \& Laurent, S. (2005). A new class of multivariate skew densities, with application to generalized autoregressive conditional heteroscedasticity models. Journal of Business \& Economic Statistics, 23(3), 346342. doi: 10.1198/073500104000000523

Bettaiah, V., \& Ranganath, H. S. (2014, March). An analysis of time series representation methods: Data mining applications perspective. In Proceedings of the 2014 ACM Southeast Regional Conference (Article 16), Kennesaw, GA. doi: $10.1145 / 2638404.2638475$

Blanchard, O. J. (1990). Why does money affect output? A survey. In B. M. Friedman \& F. H. Hahn (Eds.), Handbook of monetary economics (Vol. 2, pp. 779-835). New York: Elsevier. doi: 10.1016/S1573-4498(05)80022-0

Bollerslev, T. (1986). Generalized autoregressive conditional heteroskedasticity. Journal of Econometrics, 31, 307-327. doi: 10.1016/03044076(86)90063-1

Bollerslev, T. (1990). Modeling the coherence in short-run nominal exchange rates: A multivariate generalized arch model. Review of Economics and Statistics, 72(3), 498-505. doi: 10.2307/2109358

Campbell, J. Y., \& Ammer, J. (1993). What moves the stock and bond markets? A variance decomposition for long-term asset returns. The Journal of Finance, 48(1), 3-37. doi: 10.1111/j.1540-6261.1993.tb04700.x

Cho, M., Kim, B. H., Bae, H.-J., \& Seo, J. (2014). Stroscope: Multiscale visualization of irregularly measured time-series data. IEEE Transactions on Visualization and Computer Graphics, 20(5), 808-821. doi: 10.1109/TVCG.2013.2297933

Dambrós, N. G. G. (2014). A evolução dos mercados financeiros internacionais e os ingressos de capital para o brasil: 1995-2005 [Thesis]. Universidade Federal de Santa Catarina. Retrieved from https://repositorio.ufsc.br/handle/123456789/122040

Dornbusch, R., \& Fischer, S. (1980). Exchange rates and the current account. The American Economic Review, 70(5), 960-971. 


\section{DCC-GARCH UNDER THE BAYESIAN APPROACH}

Eichengreen, B., Rose, A. K., \& Wyplosz, C. (1996, July). Contagious currency crises (NBER Working Paper no. 5681). Cambridge, MA: National Bureau of Economic Research. doi: 10.3386/w5681

Engle, R. F. (1982). Autoregressive conditional heteroscedasticity with estimates of the variance of United Kingdom inflation. Econometrica, 50(4), 9871007. doi: $10.2307 / 1912773$

Engle, R. F. (2002). Dynamic conditional correlation: A simple class of multivariate generalized autoregressive conditional heteroskedasticity models. Journal of Business and Economic Statistics, 20(3), 339-350. doi:

10.1198/073500102288618487

Fioruci, J. A., Ehlers, R. S., \& Filho, M. G. A. (2014). Bayesian multivariate GARCH models with dynamic correlations and asymmetric error distributions. Journal of Applied Statistics, 41(2), 320-331. doi:

10.1080/02664763.2013.839635

Hansen, L. P., \& Singleton, K. J. (1983). Stochastic consumption, risk aversion, and the temporal behavior of asset returns. Journal of Political Economy, 91(2), 249-265. doi: 10.1086/261141

Heer, J., Bostock, M., \& Ogievetsky, V. (2010). A tour through the visualization zoo. Communications of the ACM, 53(6), 59-67. doi:

\section{$10.1145 / 1743546.1743567$}

Javed, W., \& Elmqvist, N. (2010). Stack zooming for multi-focus interaction in time-series data visualization. In North, S., Shen, H.-W., \& van Wijk, J. J. (Eds.), 2010 IEEE Pacific visualization symposium (pp. 33-40). Taipei, Taiwan: IEEE. doi: 10.1109/PACIFICVIS.2010.5429613

Javed, W., McDonnel, B., \& Elmqvist, N. (2010). Graphical perception of multiple time series. IEEE Transactions on Visualization and Computer Graphics 16(6), 927-934. doi: 10.1109/TVCG.2010.162

Krstajic, M., Bertini, E., \& Keim, D. A. (2011). Cloudlines: Compact display of event episodes in multiple time-series. IEEE Transactions on Visualization and Computer Graphics, 17(12), 2432-2439. doi:

\subsection{9/TVCG.2011.179}

Luo, X., Tian, F., Liu, W., Teng, D., Dai, G., \& Wang, H. (2010). Visualizing time-series data in processlines: Design and evaluation of a process enterprise application. In Proceedings of the 2010 ACM symposium on applied computing (pp. 1165-1172). New York, NY: Association for Computing Machinery. doi: 10.1145/1774088.1774336 


\section{NASCIMENTO ET AL}

Müller, W., \& Schumann, H. (2003). Visualization for modeling and simulation: visualization methods for time-dependent data - An overview. In Proceedings of the 35th conference on Winter simulation: Driving innovation (pp. 737-745). New Orleans, LA: Winter Simulation Conference. doi:

10.5555/1030818.1030916

Perin, C., Vernier, F., \& Fekete, J.-D. (2013). Interactive horizon graphs: Improving the compact visualization of multiple time series. In Proceedings of the SIGCHI conference on human factors in computing systems (pp. 3217-3226). New York, NY: Association for Computing Machinery. doi:

\section{$10.1145 / 2470654.2466441$}

Ratanamahatana, C. A., Lin, J., Gunopulos, D., Keogh, E. J., Vlachos, M., \& Das, G. (2005). Mining time series data. In O. Maimon \& L. Rokach, (Eds.), The Data mining and knowledge discovery handbook (pp. 1069-1103). Boston, MA: Springer. doi: 10.1007/0-387-25465-X_51

Shneiderman, B. (2008). Extreme visualization: Squeezing a billion records into a million pixels. In Proceedings of the 2008 ACM SIGMOD international conference on management of data (pp. 3-12). New York, NY: Association for Computing Machinery. doi: 10.1145/1376616.1376618

Telea, A. C. (2014). Data visualization: Principles and practice (2nd edition). CRC Press. doi: 10.1201/b17217

Tse, Y. K., \& Tsui, A. K. C. (2002). A multivariate generalized autoregressive conditional heteroscedasticity model with time-varying correlations. Journal of Business and Economic Statistics, 20(3), 351-362. doi: 10.1198/073500102288618496 